\title{
La comunicación de experiencias de marca a través de las redes sociales: análisis de caso de los patrocinadores del Mundial de Fútbol 2010
}

\author{
Silvia GARCÍA MIRÓN ${ }^{1}$ \\ Universidade de Vigo
}

\begin{abstract}
RESUMEN:
Las agencias de publicidad se están viendo afectadas en su planificación diaria y en la creación de campañas por grandes cambios que han llegado de la mano de la implantación de las NTICs. Esto implica que se vean modificadas las estrategias y objetivos de los anunciantes e incluso la forma de plantearse su rutina de trabajo. De entre todas las técnicas y fórmulas comunicativas que han surgido con Internet, las redes sociales están siendo las que más posibilidades ofrecen no solo a cualquier tipo de usuario, sino a los medios de comunicación y, evidentemente, a cualquier anunciante, ya sea con un carácter más local o internacional, para conseguir una mayor cercanía con sus consumidores y obtener información más precisa de sus actitudes, gustos e intereses. De este modo, podemos observar cómo los anunciantes utilizan dichas redes sociales en la búsqueda de una mayor complicidad y fidelización con segmentos concretos de su target. En este artículo, demostraremos esta presencia en redes sociales a través de las campañas basadas en brand experiences por parte de los patrocinadores y colaboradores del Mundial de Fútbol de Sudáfrica 2010 junto con los patrocinadores de la Selección Española de Fútbol, estudiando sus actuaciones en la red social Facebook durante dicho evento deportivo.
\end{abstract}

PALABRAS CLAVE: Redes sociales; experiencia de marca; estrategia; anunciante; Copa del Mundo de la FIFA 2010.

TITLE: Brand experiences communication in social networks: case analyis of FIFA World Cup 2010 sponsors

\begin{abstract}
:
Advertising agencies are being influenced in their routine planning and in their campaign creation by huge changes which have come with the NCITs. This supposed that announcer's strategies and objectives are being modified, as well as the way of thinking about their working routine. Among all of the different communicative techniques and formulas arised with the Internet, social networks are being those which can offer more possibilities not only for users, but also for mass media and, obviously, for every announcer, with local or international nature, in order to get a closer relation with its consumers as well as more specific information about their attitudes, tastes and interests. Hence, we can see how announcers use these social networks in search of more proximity and fidelization with special spectator segments. In this paper, we will prove this presence in social networks through cam-

1 Silvia García Mirón es profesora de la Facultad de Ciencias Sociales y de la Comunicación de la Universidad de Vigo en el grado en Publicidad y Relaciones Públicas. Ha publicado varios artículos y comunicaciones centrándose principalmente en el ámbito televisivo (programación, identidad de canal, sinergia de la televisión con otros medios, la publicidad en televisión, etc.) y también ha investigado sobre la adaptación de materias de comunicación al EEES a través de la innovación en la docencia universitaria. Su tesis doctoral, en proceso de elaboración, se centra en un análisis acerca de la identidad de canal y la programación de Antena 3. Su correo electrónico es: silviamiron@uvigo.es
\end{abstract}


paigns based on brand experiences by the sponsors and contributors of the FIFA World Cup 2010 as well as the Spanish team sponsors, analysing their actions in the social network Facebook for this event.

KEY WORDS: Social Networks; brand experiences; strategy; announcer; FIFA World Cup 2010

\section{Introducción y aspectos metodológicos}

El ámbito de la comunicación está experimentando cambios a paso de gigante, consiguiendo nuevos medios y soportes, inventando nuevas fórmulas, descubriendo nuevas tácticas, etc. y, de esta forma, las agencias de publicidad y Relaciones Públicas deben involucrarse y no dejar escapar ni una oportunidad de conseguir diferenciación para las campañas de sus clientes, aplicando todo aquello que sea novedoso y que les produzca algún tipo de beneficio (ya sea de carácter económico, social o en términos de imagen) y que permita que la campaña de que se trate sea eficaz.

La creación de experiencias de marca no es algo nuevo. En realidad, desde siempre el consumidor ha experimentado un proceso sensorial con la prueba, uso o disfrute de un producto o servicio. No obstante, no será hasta los años ochenta cuando la perspectiva marquetiniana comience a vislumbrar un nuevo rendimiento a estas experiencias, un beneficio que puede repercutir favorablemente sobre la imagen de la marca. Hoy en día, las brand experiences (o experiencias de marca) se configuran como una de las estrategias clave de los anunciantes, empleando todos los medios y herramientas posibles para hacer de este elemento identitario una fórmula con la que conseguir un mayor acercamiento al consumidor. Asimismo, resulta relevante señalar cómo el desarrollo tecnológico ha conseguido que estas experiencias supongan una ventaja competitiva y diferencial para el anunciante. El empleo de la tecnología aplicado a las brand experiences puede ser diverso, pero lo que está fuera de duda es la utilización de las redes sociales para comunicar lo que se puede conseguir en este sentido. Una herramienta de este tipo se convierte no solo en una técnica comunicativa más, sino en un soporte promocional y publicitario de primer orden ${ }^{2}$.

La Red se ha convertido en una fuente de información imprescindible y un medio con un número de usuarios que va en aumento cada día que pasa, al igual que sucede con los seguidores de las redes sociales. De acuerdo con el estudio «Navegantes en la Red» realizado por la $\mathrm{AIMC}^{3}$, obtenemos que ya sólo el $28,4 \%$ de los

2 Esta importancia se traslada a ciertos datos relevantes, tales como que la agencia DDB ya haya lanzado una división llamada Radar DDB especializada en redes sociales y en nuevas formas de establecer relaciones continuas con el consumidor mediante plataformas como Twitter o Facebook; o que McDonald's haya nombrado a un director de redes sociales dentro de su estructura organizativa.

Cfr. Aimc-Asociación Para La Investigación De Los Medios De Comunicación (2010): Navegantes en la Red. $12^{a}$ encuesta AIMC a usuarios de Internet, Madrid, Sersa. 
usuarios no está registrado en ninguna red $\operatorname{social}^{4} \mathrm{y}$ también se demuestra la importancia que ha adquirido Internet para consultar opiniones de otros consumidores sobre un producto 5 .

Teniendo en cuenta las ideas presentadas, nos planteamos con esta investigación indagar en la relación existente entre redes sociales y comunicación de experiencias de marca de un grupo de marcas unidas por un elemento común: su participación como anunciantes en el Campeonato Mundial de Fútbol de 2010, observando el comportamiento de estos patrocinadores y la participación del usuario a través de la red social. Como caso de análisis nos centramos en los patrocinadores, socios colaboradores y colaboradores oficiales de la FIFA y del Mundial de Sudáfrica 2010, junto con los patrocinadores de la Selección Española de Fútbol durante este campeonato. Desde nuestra perspectiva, interesa observar el comportamiento que distintas marcas anunciantes, de mayor o menor relevancia publicitaria en niveles de inversión y de sectores de consumo también diversos, llevan a cabo en los nuevos soportes y ámbitos comunicativos que surgen gracias al desarrollo de las Nuevas Tecnologías de la Comunicación y de la Información, NTICs de ahora en adelante.

En cuanto a red social, hemos delimitado la muestra a Facebook, por ser la herramienta que cuenta con un mayor número de usuarios tanto en España como a nivel internacional ${ }^{6}$, muy por encima de otras redes sociales como la española Tuenti, Twitter o Hi5, que además no permiten estrategias empresariales a la altura de las posibilidades que ofrece Facebook.

El contexto es uno de los aspectos clave para esta investigación, ya que «el significado y la experiencia de marca que obtiene el usuario es totalmente dependiente del contexto en el que se hayan inscritas» ${ }^{7}$. Para el caso propuesto, dicho contexto vendrá delimitado por el Campeonato Mundial de Fútbol, un evento de gran relevancia no sólo deportiva sino también publicitaria.

Con las ideas principales sobre la mesa, señalaremos las hipótesis de partida que establecemos para la presente investigación:

4 Los datos presentados se corresponden con el año 2009, pues los últimos resultados publicados son del mes de Febrero del 2010. No obstante, todas las señales y estadísticas publicadas por las propias redes sociales demuestran que el número de usuarios continúa en aumento.

5 En relación con la influencia de las opiniones generadas en Internet por los propios consumidores, nos encontramos con que el $69 \%$ de los usuarios ha utilizado la Red en los últimos treinta días para consultar opiniones de otras personas acerca de distintos productos o servicios y un $43,2 \%$ confía en la veracidad de la información consultada. Por otra parte, más del 30\% ha colgado algún comentario en Internet dando su opinión sobre determinados productos. Todos ellos son datos significativos que nos hacen darnos cuenta de la fuerza de Internet para influenciar la imagen de una marca a través de los propios usuarios, de sus palabras y de sus actuaciones en este medio.

6 Las últimas cifras publicadas señalan que la red social Facebook cuenta con más de quinientos millones de usuarios activos. Cfr. Sala de prensa de la red social Facebook en http://www.facebook.com/press/info.php?statistics. Web visitada el 30/10/2010.

7 ROYO, J.: «Identidad líquida. Las marcas en el ciberespacio», http://www.joancosta.com/identidad_liquida.htm. Web visitada el 02/11/2010.

Joancosta.com, 
- Los anunciantes actuales, ante un evento concreto y significativo publicitariamente hablando (como es el caso de un Campeonato Mundial de Fútbol), del que pueden verse beneficiados no solo económicamente sino también a niveles de imagen, apuestan por utilizar estrategias en las que se vea implicado su consumidor: es decir, estrategias basadas en las brand experiences.

- Una de las funciones de las redes sociales, por parte de los anunciantes, consiste en comunicar el desarrollo de su experiencia de marca.

- Los seguidores de la marca se interesan por este tipo de experiencia y lo demuestran con su participación e interacción a través de su perfil y sus publicaciones en la red social.

- Este tipo de campañas tienen como objetivo la búsqueda de participación del consumidor y una respuesta de tipo emocional.

- En consecuencia, las redes sociales, especialmente Facebook, se convierten en una de las principales presencias de los anunciantes en Internet.

- Los anunciantes, durante un evento de tipo deportivo - como es el caso de un Mundial de Fútbol- apuestan por experiencias de marca que muestren una evolución a la par que se produce el desarrollo del evento.

La metodología que seguiremos se basará, en primer lugar, en un estudio bibliográfico sobre los dos ámbitos unidos en este artículo, a saber, las redes sociales y las experiencias de marca, y por otra parte, emplearemos la observación y el análisis de contenido. Estudiaremos, en una etapa inicial, la presencia en la red social Facebook de los patrocinadores y colaboradores de la FIFA, el Mundial y de la Selección Española de Fútbol, analizando las características de dicha presencia. En una segunda fase de tipo exploratoria, recogeremos las entradas realizadas por cada uno de los patrocinadores y colaboradores con presencia en Facebook durante un mes. A partir de aquí, y en una fase interpretativa, podremos descubrir, si realmente se dio el caso, la creación de experiencias de marca con los anunciantes que conforman la muestra y, en caso afirmativo, realizar un análisis de dichas experiencias, de la comunicación a través de la red social y delimitar la implicación que se obtuvo por parte de los usuarios y seguidores del perfil en Facebook de estas marcas.

La observación se contempla como la mejor de las fórmulas que podemos emplear, pues comprendemos que esta metodología es la óptima para determinar los cambios producidos en el consumo mediático, en el contexto delimitado por la Sociedad de la Comunicación. 


\section{Acercamiento al concepto de red social}

\subsection{Las redes sociales como concepto A.I. (Antes de Internet)}

El concepto de red social surge en un ámbito diferente del que se ocupa este estudio: un entorno que en su momento desconocía lo que podían suponer para las relaciones humanas las técnicas que se desarrollarían a través de las NTICs. Porque si las redes a las que nos referimos son las que forman las personas al relacionarse las unas con las otras, podríamos decir entonces que la sociedad siempre ha sido una red.

En el ámbito de la sociología, una red social puede ser entendida -de acuerdo con la imagen metafórica de red- como «una serie compleja de interrelaciones dentro de un sistema social» ${ }^{8}$. No obstante, Requena Santos aclara este concepto para su estudio, desde una perspectiva analítica, a través de la siguiente explicación del término, que él entiende como:

[...] un conjunto de puntos (actores sociales) vinculados por una serie de relaciones que cumplen determinadas propiedades. Las redes sociales gozan de una estructura y una morfología propias, cuyas cualidades, como la posibilidad de cuantificar las relaciones y su consiguiente tratamiento matemático, evidencian importantes aplicaciones para el análisis e interpretación de las conductas sociales 9

Simplificando esta idea, diríamos que vivimos en un sistema e interactuamos con él, adaptándonos a las nuevas circunstancias que vayan influenciando este sistema $^{10}$. Es por eso que, «hoy por hoy, Facebook es un fenómeno social, del cual muy poca gente puede abstraerse, aún sin comprender exactamente cuál es su utilidad. Porque Facebook - mal que nos pese- es un simulador de la sociedad (una sociedad de clase media/media alta, con acceso a Internet y preocupaciones más o menos burguesas)» ${ }^{11}$.

Asimismo, si partimos de la premisa de que la naturaleza humana anida en la naturaleza social -pues, tal y como afirmaba el filósofo Aristóteles, «El hombre es un animal social»-, podremos entender muchos de los comportamientos humanos y su evolución a lo largo de la historia del hombre, hasta el paso que se ha dado te-

8 REQUENA SANTOS, F. (1989): «El concepto de red social», Revista de Investigaciones SociológicasREIS, 48, 138.

9 Ibidem, 137.

10 Si bien en el lenguaje cotidiano se ha utilizado este concepto para hacer referencia a conjuntos complejos de relaciones entre miembros de sistemas sociales, no será hasta 1954 cuando J. A. Barnes, antropólogo de la Escuela de Manchester, comience a utilizar dicho término de forma sistemática para mostrar patrones de lazos, incluyendo conceptos tradicionalmente utilizados por los científicos sociales, como es el caso de «grupos sociales» o «categorías sociales».

11 FAERman, J. (2010): Faceboom. Facebook, el nuevo fenómeno de masas, Barcelona, Ediciones B Argentina y Grupo Planeta, 18. 
niendo en cuenta la influencia de los avances tecnológicos en la conducta humana. Porque, para hablar de redes sociales hoy en día, es preciso hacer referencia a dos elementos que no se tenían en consideración en la definición de Requena Santos citada y que han sido propuestos por Ugarte $^{12}$ : por una parte Internet y su consecuencia más directa, la eclosión de una nueva esfera de relación social que pone en contacto a millones de personas cada día; y, por otra parte, la aparición en los últimos años de una amplia literatura sobre redes aplicada a todos los campos.

Ese último paso en la evolución del ser humano se produce en base a las nuevas redes sociales que surgen a través de Internet, como es el caso de MySpace, Facebook o Hi5. De acuerdo con las ideas que muestran Christakis y Fowler, las redes sociales, las de siempre, y las ciberespaciales, son un superorganismo vivo, aunque ofrecen una diferencia fundamental entre ellas, ya que «una de las características que distinguen a los mundos virtuales del real es nuestra capacidad de controlar nuestra presencia.... ${ }^{13}$.

Importante resulta también aclarar el concepto de «sociedad», caracterizado siguiendo las teorías del sociólogo Georg Simmel ${ }^{14}$ - por las acciones recíprocas en las que entran varios individuos.

Esta acción recíproca se produce siempre por determinados instintos o para determinados fines. Instintos eróticos, religiosos o simplemente sociales, fines de defensa o de ataque, de juego o adquisición, de ayuda o enseñanza, e infinitos otros, hacen que el hombre se ponga en convivencia, en acción conjunta, en correlación de circunstancias con otros hombres; es decir, que ejerza influencias sobre ellos y a su vez las reciba de ellos. La existencia de estas acciones recíprocas significa que los portadores individuales de aquellos instintos y fines, que los movieron a unirse, se han convertido en una unidad, en una sociedad ${ }^{15}$.

Estas acciones recíprocas son las que están presentes también en las redes sociales virtuales. Podríamos, por tanto, concluir que Facebook es una herramienta de socialización, al tiempo que de comunicación, que garantiza el intercambio de instintos o fines entre varios individuos.

12 Cfr. Ugarte, D. (2007): El poder de las redes. Manual ilustrado para personas, colectivos y empresas abocados al ciberctivismo, Barcelona, El Cobre Ediciones, 23.

13 Christakis, N. A.; Fowler, J. H. (2010): Conectados, Madrid, Taurus, 21.

14 Georg Simmel fue uno de los primeros estudiosos en analizar las redes sociales, especialmente desde la perspectiva de la naturaleza del tamaño de la red sobre la interacción y la probabilidad de interacción en redes, en lugar de grupos.

15 Simmel, G. (1986): Sociología, 1. Estudios sobre las formas de socialización, Madrid, Alianza Editorial, 15-16. 


\subsection{Posibilidades comunicativas y publicitarias de las redes sociales: algunos datos significativos sobre Facebook}

El desarrollo de aplicaciones que simulan una red social real en internet ha conseguido que se desenvuelva su uso con fines estratégicos empresariales. Las redes sociales virtuales no solo facilitan la comunicación entre sus usuarios, sino que gracias a ellas las empresas pueden establecer otro tipo de comunicación, más cercana, con sus clientes o con los usuarios de los servicios que ofrecen.

En la búsqueda por mantener las mejores opciones de contacto entre los individuos que las habitan, las redes sociales incluyen opciones de interactividad. Esto afecta positivamente a las acciones y tareas que puede llevar a cabo una empresa al conseguir involucrar a sus clientes. Éste deja de ser un consumidor pasivo de los contenidos para convertirse, no ya en un ser activo, sino en un "prosumidor», un consumidor que produce contenidos, que comenta las noticias e informaciones relacionadas con la empresa o la marca en la red social, y que incluso puede informar él mismo de aquellas noticias que conoce al respecto. Este tipo de actuaciones comenzaron en los albores de la Web $2.0^{16}$, concepto $^{17}$ que esconde, al mismo tiempo, toda una serie de aplicaciones y servicios cuya lógica es, en ocasiones, justamente la opuesta. En vez de generar abundancia, generan escasez mediante la formación de un único output igual para todos los usuarios ${ }^{18}$. No hay segmentación de los mensajes a quienes va dirigido. Este tipo de actuaciones segmentadas únicamente puede observarse en la creación de grupos o comunidades concretas.

La idea expuesta se puede explicar, en parte, a través del concepto groundswell $^{19}$, «un fenómeno social que consiste en que las personas utilizan las tecnologías para intercambiarse lo que necesitan, sin recurrir a entidades tradicionales como las empresas ${ }^{20}$. Gracias a la tecnología que se ha desarrollado, junto con la fuerza que pueden llegar a ejercer millones de personas, las empresas pueden reorientar sus estrategias con vistas a hacer uso de esta fuerza en su provecho para facilitar la difusión de aquellos mensajes que necesiten enviar a todos sus clientes. Una fórmula de comunicación que implica dejar en manos de sus clientes el mensaje, con lo que ello supone; es decir, adquiere la virtud de mayor credibili-

16 Término acuñado por el analista de Internet Tim O'Reilly.

17 Los inicios de la Web 2.0 tuvieron lugar con determinados portales como Flickr o YouTube, que prestaban gratuitamente las herramientas a los usuarios y generaban espacio en sus propios servidores. $\mathrm{Al}$ permitir que cualquier navegante suba cualquier tipo de contenido y permitir asimismo que todos esos contenidos estén a disposición de todos los usuarios, podríamos señalar que la selección depende de los individuos y no de las propias páginas.

18 Cfr. UGarte, op. cit., 110.

19 El portal de ventas eBay, el sistema operativo Linux, la página BitTorrent para compartir música, etc.; todos ellos son ejemplos de lo que significa groundswell, una forma distinta de relación entre individuos y entre empresas y personas.

20 Li, C.; BeRnOFF, J. (2008): El mundo Groundswell. Cómo aprovechar los movimientos sociales espontáneos en la Red, Barcelona, Ediciones Urano, 30. 
dad (cuando un cliente consigue la opinión o el mensaje directamente de otro cliente igual a él), pero también la desventaja de perder el control sobre dicho mensaje y que este llegue incluso a transformarse en algo negativo para la imagen de la empresa.

La ventaja diferencial de las redes sociales con respecto a otras técnicas empleadas a través de Internet reside principalmente en la opción de poder interactuar con los consumidores consiguiendo una relación más cercana. Así lo entiende Yolanda Vázquez, coordinadora de la División Digital EuroRSCG Buenos Aires ${ }^{21}$ :

La posibilidad de crear espacios en abierto para que los usuarios interactúen entre ellos es su principal ventaja. Antes las agencias disponíamos únicamente de los tradicionales foros, que todavía se utilizan, pero su dinámica es otra, básicamente como un modo de resolución de dudas. En las redes sociales, sin embargo, la marca, si lo hace bien, puede ser un usuario más, un amigo más.

Gracias a este tipo de beneficios comunicativos, los anunciantes demandan cada vez más a sus agencias la inclusión de redes sociales no solo en su planificación de medios, sino también en el diseño de sus estrategias creativas para poder orientar sus campañas desde los social media.

Son numerosas las redes sociales disponibles en el mundo virtual a día de hoy. Pero, entre todas ellas, Facebook es, como ya hemos visto, la que sobresale por número de usuario/as a nivel nacional e internacional, así como la más notoria y la que mejor funciona para mantener el contacto con los amigos ${ }^{22}$. Fundada en febrero de 2004, la mayoría de sus funciones se basa en la idea de que hay personas en nuestras vidas con las que queremos mantener contacto, ya sean amigos, familiares, compañeros de trabajo o simplemente conocidos. No obstante, existen autores o usuarios que realmente ponen en duda estas capacidades de socialización, pues, si bien permite acercar a los que están lejos, también puede provocar que se alejen los que están cerca. «Y, en muchos casos, en vez de favorecer la comunicación, la limitan», tal y como afirma Faerman ${ }^{23}$, quien insiste en que la tecnología en ocasiones «trae como tendencia el aislamiento social, más que la tan declamada integración» $\rangle^{24}$. Lo cierto es que Facebook ha conseguido desbancar las llamadas de teléfono o incluso los correos electrónicos simplemente por un vistazo al «muro» de aquel de quien queremos saber.

Sus miembros mantienen perfiles con aquella información personal que deseen dar a conocer, publican noticias o sus «estados», llevan a cabo invitaciones a even-

\footnotetext{
21 Entrevista personal realizada por la autora, el día 28/04/2010.

22 Estos datos han sigo publicados por Elogia Ipsofacto de acuerdo con un estudio en colaboración con el Departamento de Investigación del Interactive Advertising Bureau Spain. http://www.slideshare.net/IAB Spain/informe-sobre-redes-sociales-en-espaa. Web visitada el 20/10/2010.

23 FAERMAN, op. cit, 25.

24 Idem, p.60.
} 
tos, tienen la posibilidad de incluir álbumes de fotos y sus miembros pueden conectar e interactuar entre sí. De todo esto, la actividad que más significancia posee, y la que da nombre a estas herramientas, es el «friending», mecanismo por el cual los miembros de Facebook agregan a su perfil a amigos o conocidos y mantienen el contacto entre ellos, en función de un determinado interés o finalidad de cualquier tipo, creando así vínculos para cohabitar en esta sociedad virtual. No obstante, las redes sociales, especialmente Facebook, se están convirtiendo en plataformas que ofrecen miniaplicaciones de juegos o cuestiones de lo más variado, lo que ayuda a ampliar el campo de actividades que los amigos pueden realizar de forma conjunta.

De acuerdo con la información estadística ${ }^{25}$ que ofrece esta herramienta social, aproximadamente el $50 \%$ de sus 500 millones de usuarios entra cada día. El usuario medio tiene unos 130 amigos, está conectado a ochenta comunidades, grupos o eventos (existen más de novecientos millones de elementos con los que los usuarios de la red social pueden interactuar: páginas, eventos, grupos, etc.) y publica unas noventa piezas de contenido cada mes, lo que conlleva que se compartan más de treinta billones de publicaciones cada mes (estados, enlaces, fotos, etc.).

Por todos los datos ofrecidos, la presencia de una empresa hoy en día en redes sociales ya es un hecho, incluyéndose en la planificación publicitaria de las cuentas de los clientes en las agencias. Así se demuestra en el Estudio de Agencias (Estudio de Imagen de las Agencias de Publicidad en España), donde se destaca como una de las nuevas tareas que realizan las agencias la respuesta directa (antes del 2006 todavía no se incluía), como también en el significativo aumento del marketing interactivo ${ }^{26}$ o en la creación de nuevos perfiles profesionales, como es el caso de los comunity managers.

\section{Las experiencias de marca como nuevo concepto publicitario de éxito: la participación como reto}

\subsection{Introducción al concepto "experiencia de marca»}

Cuando se habla de marcas lo primero en que piensa el consumidor es en su nombre y su logotipo, pero también, cada vez más, en las experiencias de marca que puede conseguir al disfrutar de un determinado producto. Cuando hablamos de experiencia de marca no nos referimos únicamente a la oferta de un servicio al consumidor, ya que cualquier producto puede generar experiencias de marca inol-

25 Cfr. Sala de prensa de la red social Facebook, en http://www.facebook.com/press/info.php?statistics. Web visitada el 30/10/2010.

26 AsOCIACIÓN ESPAÑOla DE AgENCIAS DE PUBLICIDAD: «Estudio de Agencias 2008. Estudio de Imagen de las Agencias de Publicidad en España» (versión resumen online). http://www.aeap.es/archivo/e01816537c5196080e1c3ee181130eae.pdf. Web visitada el 15/11/2010. 
vidables para su público. Fueron precisamente Pine y Gilmore ${ }^{27}$ quienes separaron experiencia y servicio, señalando que la creación de experiencia para el cliente no se limitaba a las empresas de servicios o a una función de servicios, ya que ciertos negocios exitosos -como el parque temático de Walt Disney- ofrecían más que el consumo de un producto: generaban experiencias inolvidables. Estos autores, reconocidos por llevar la experiencia al primer plano del marketing empresarial, demuestran que lo que la gente busca hoy en día son experiencias, definidas por ellos como «eventos que comprometen a los individuos de una forma personal» y demuestran que ofrecer experiencias en el mercado actual es un buen negocio. Así lo afirman LaSalle y Britton, al señalar que «explicando la progresión del valor económico de bienes y servicios y en última instancia la experiencia, demuestran que el valor de una oferta que incluya factores como la diversión o el entretenimiento es significativamente mayor que aquellos que no tienen estos atributos ${ }^{28}$.

Sin embargo, ya anteriormente, en 1982, Holbrook y Hirschman ${ }^{29}$ introdujeron la idea de que la experiencia del cliente podría ser considerada por sus propios méritos, abogando por la idea del consumo a través del placer. «Este tipo de consumo busca diversión, entretenimiento, fantasía, interés y estímulo sensorial ${ }^{30}$. Esta filosofía de pensamiento en el ámbito marquetiniano dio paso a que otro autor, Bernd H. Schmitt, siguiese la línea de la experiencia sensorial en el consumo de las marcas $^{31}$.

No cabe duda de que la marca va más allá de su nombre y símbolo. Es la síntesis de una propuesta de creación de valor a través de numerosos aspectos dependientes de la marca o con los que interactúa. La experiencia de marca tiene como componentes «a la marca en sí misma, la forma en que ella se comunica, el lugar donde está presente, los servicios asociados al producto que se ofrece, la disponibilidad del producto y/o servicio, y el valor y la utilidad que se la da al mismo» ${ }^{32}$.

La experiencia de marca procede, como enfoque teórico, de la creación de marca a través de la personalidad de la misma, otorgándole valores y sentimientos con la intencionalidad última de conseguir una ventaja diferencial en este nivel, crean-

27 PINE, B. J.; GILMORE, J. H. (1999): The Experience Economy: Work is theatre and every Business a stage, Boston, Harvard Business School Press.

28 LASALle, D.; BRITTON, T. A. (2006): Cómo añadir valor a sus productos y servicios: convertir los productos ordinarios en experiencias extraordinarias, Barcelona, Deusto, 9.

29 HolbroOK, M. B.; HIRSCHMAN, E. C. (1982): «The experiential aspects of consumption: consumer fantasies, feelings and fun», The Journal of Consumer Research, 9(2), Chicago, The University of Chicago Press, 132-140.

Ibidem, 134.

31 Como se expresa en Sснмiтt, B. (1999): Experiential Marketing: how to get customers to sense, feel, think, act and relate to your company and brands, Nueva York, The Free Press.

32 SARACCO, C. «La experiencia de marca va más allá de un símbolo», Business Opportunities, http://espanol.business-opportunities.biz/2006/02/28/la-experiencia-de-marca-va-mas-alla-de-un-simbolo/. Web visitada el 27/10/2010. 
do una imagen concreta ${ }^{33}$, con físico y forma de ser. Pero lo más importante es que una experiencia de marca parte de la interacción entre un cliente y una marca, por lo que nunca se producirá sin la participación del consumidor. La participación, por tanto, será la clave para entender este concepto en la actualidad.

\subsection{El planteamiento de la experiencia de marca: la aplicación de los cinco sentidos}

En la creación de una campaña de este tipo se seguirán una serie de pasos que no difieren demasiado de los pasos que se han de seguir en cualquier otro tipo de campaña. La principal diferencia radica en la meta que se pretende conseguir, en su planteamiento: la implicación que se requiere por parte del consumidor. El primer paso consistirá en reconocer cuáles son las audiencias clave de la empresa, ya sean éstas internas o externas, y realizar una investigación sobre sus comportamientos, actitudes e intereses para buscar la experiencia de marca más acorde con el segmento de público al que la marca desea dirigirse. El segundo paso radicará en revisar la promesa de la marca y valorar si se puede plantear una experiencia a través de las cualidades y valores, sin olvidarnos de su posicionamiento en el mercado. Estos valores se establecerán en función de su cultura corporativa, su comportamiento organizacional, sus políticas de servicio, sus políticas de marketing, etc. y, especialmente, su personalidad como marca. La promesa debe orientarse hacia la búsqueda de complicidad con el consumidor, hacia la integración del público en el mundo y los valores de la mar$\mathrm{ca}$, a hacer que este se sienta importante e incluso protagonista para la marca. A continuación, debe describirse detalladamente la experiencia de marca junto con el plan de acción, relacionándola con emociones concretas que se pretendan conseguir en el consumidor. A partir de aquí, debe plantearse un seguimiento y una evaluación posterior de dicha experiencia de marca, al igual que haríamos con cualquier otra campaña publicitaria o de comunicación.

De todo lo comentado en este epígrafe se desprende que las agencias de publicidad abogan por sustituir el concepto de imagen de marca por el de experiencia de marca, siendo lo importante su aplicación a la marcas reales y no solo su presencia en diversos manuales teóricos ${ }^{34}$.

El auge de las experiencias de marca ha conducido a que se hayan desarrollado ciertos estudios centrados en este tema, como el realizado por la agencia de marketing estratégico y branding Allegro 234, la cual se ha encargado de la construcción de

33 Costa incide en el tema de la imagen de las marcas, señalando que la imagen pone dos mundos en interacción: el físico y el mental, psicológico y cultural. La imagen se encontraría en este segundo mundo, con las percepciones y las experiencias. Cfr. CosTA, J. (2004): La imagen de marca, Barcelona, Paidós.

34 Tal es el caso de Brand sense. Convierta su marca en una experiencia de cinco sentidos, de Martin Lindstrom, o el citado Experiential Marketing, de Bernd H. Schmitt. 
un ranking de las experiencias más atractivas denominado «Coolest \& Gaps»35. Dicho estudio concluye con dos ideas esenciales: que la comunicación es solo una parte de la experiencia y que las experiencias de marca más cool son aquellas que se viven con los cinco sentidos. Para Lindstrom el secreto radica en la creación de una marca multisensorial, pues se conseguirá un efecto publicitario mayor, y señala:

[...] esta sinergia sensorial total produce un efecto dominó. Debido a la forma en que las impresiones se almacenan en el cerebro, si usted desencadena uno de los sentidos, ése llevará al siguiente, y después al siguiente... y la consecuencia es que una visión total de recuerdos y emociones se revelará al instante. Tener éxito con dos elementos solo es la mitad de la historia; la última meta debe ser integrar todos los sentidos ${ }^{36}$.

La gestión de marcas siempre ha consistido en establecer vínculos emocionales entre la marca y el consumidor. De hecho, «puede afirmarse que optimiza el comportamiento de compra por impulso, provoca nuestro interés y permite que nuestra respuesta emocional predomine sobre nuestro pensamiento racional» ${ }^{37}$.

\subsection{Principales tácticas de las experiencias de marca: espacios arquitectónicos y desarrollo de tipo tecnológico}

Una de las opciones de transmisión de experiencia de marca que han tomado un repunte en los últimos años es el desarrollo arquitectónico preparado por y para la marca. Hablamos de la innovación en los puntos de venta a través de la creación de recintos especiales para el uso y disfrute de los consumidores donde solo tendrá presencia una única marca que desea así convertirse en protagonista y dedicarse por completo a su público. Desde las flagship stores -la tienda más importante, el buque insignia de la firma que consigue trasladar un valor añadido a los productos de la marca-, quizás más arraigadas en la idea tradicional de tienda o centro comercial de una marca relacionada con la moda o el mundo deportivo ${ }^{38}$, a ideas más innovadoras que vienen de la mano de la introducción en el ámbito de la hostelería:

35 En función de esto, las cinco marcas que ofrecerían una experiencia más satisfactoria para el consumidor serían: Apple (con más del doble de menciones por parte de los encuestados que el segundo), Coca Cola, Disney, Ferrari y Virgin. Cfr. ALLEGRo 234: «Los resultados del Coolest \& Gaps Branding Survey», Allegro 234, http://www.allegro234.net/noticia.asp?vcnoticia=14. Web visitada el 01/11/2010.

36 LindSTROM, M. (2007): Brand sense. Convierta su marca en una experiencia de cinco sentidos, México, Grupo Editorial Patria, 33.

37 LINDSTROM, op. cit, 105.

38 La marca Nike presentó su nuevo flagship store en Tokio. La idea de Nike consistió en volcar todo el universo de la marca en un espacio de casi $1000 \mathrm{~m}^{2}$ donde la diferencia radica en el desarrollo del primer NikeID de Japón, que permite a los consumidores crear sus propias zapatillas y customizarlas según sus preferencias. Cfr. «Cómo transmitir la experiencia de marca a través de la arquitectura», Espacioliving, http://www.espacioliving.com/nota.asp?nota_id=1223734. Web visitada el 28/10/2010. 
la creación de cafés (Gucci en Milán) u hoteles (Bulgari también en Milán, Miss Sixty en Riccione o Camper en el barrio de El Raval de Barcelona ${ }^{39}$ ), las Nutellerías o las tiendas/bar nutricionales de Danone, recintos de prueba y degustación de un producto donde los únicos elementos que rodean al consumidor pertenecen a la simbología de la propia marca, sin despistes para el consumidor con otros logotipos de marcas pertenecientes a su competencia ${ }^{40}$. Se trata, en todos estos casos, del traslado de los valores de la marca a un espacio arquitectónico propio.

Otra de las opciones proviene de la mano de la tecnología. La experiencia de marca a través de las webs se concibe como otro punto de contacto entre consumidor y marca que tiene que ser planificado y desarrollado correctamente de acuerdo con la transmisión de valores de la marca. El diseño visual, el diseño de interacción, la arquitectura del site, etc., todo ello influirá de forma determinante en la relación consumidor-marca y esta experiencia también contribuirá a la creación de imagen de marca. No obstante, parece que últimamente la tendencia consiste en la creación y comunicación de experiencias de marca a través de las redes sociales. Facebook o Twitter de forma predominante son el vehículo básico de transmisión de todo el proceso que se puede llevar a cabo con una experiencia de marca innovadora. Mientras otro tipo de técnicas comunicativas como la publicidad sirven para comunicar el inicio del evento o sus resultados, las redes sociales son la herramienta fundamental de diálogo entre la marca y los consumidores.

Este discurso, esta narrativa, estas construcciones son la base de la experiencia de marca. La ubicuidad de la marca es una realidad gracias al desarrollo tecnológico, y esta ubicuidad permite que sea el consumidor el que acceda a la marca por sí mismo cuando lo necesita. Siguiendo a Montero, la experiencia de marca supone la eclosión de las comunicaciones pull, puesto que el consumidor ya está lo suficientemente educado como para tomar la iniciativa en sus relaciones con la marca. «Lo máximo a lo que puede aspirar una marca hoy en día es a trasladar todo el peso de la misma a sus clientes. Convertirlos en soporte publicitario, convertirlos en los constructores del posicionamiento, convertirlos en los vigilantes de su integridad.... ${ }^{41}$. Todo esto conduce a nuevos paradigmas comunicativos: la comunicación

39 El reto autoimpuesto por Camper ha consistido en trasladar a un hotel urbano los principios que diferencian su producto básico: la oferta de un establecimiento tranquilo y relajado en el que el usuario encuentre comodidad para disfrutar de la experiencia de marca Camper. Cfr. Página web corporativa de Camper, http://www.casacamper.com/barcelona/default-es.htm! .Web visitada el 28/10/2010.

40 Martin Lindstrom (2007) destaca como la primera experiencia sensorial la «Singapore girl», campaña que cambió la imagen de la Singapore Airlines a partir de 1973. La compañía aérea innovó y se diferenció de sus competidores cuando lanzó una campaña centrada en la experiencia emocional de viajar por aire frente a mostrar únicamente en sus anteriores promociones valores ciertamente más tangibles como el diseño de la cabina, la comida, el confort o los precios.

41 Montero, L.: «La experiencia de marca», Baquia, negocios y Nuevas Tecnologías, http://www.baquia.com/com//20021211/art00011.html. Web visitada el 20/10/2010. 
crossmedia $^{42}$, pues han aumentado las formas de acceso a la marca así como los momentos en los que el consumidor desea tener contacto con aquélla.

No obstante, lo que no debemos olvidar es que esta experiencia de marca debe, finalmente, enfocarse hacia una repercusión positiva para la marca y, por ende, para el anunciante: es decir, beneficios de tipo económico o de imagen que se conviertan en generación de valor como principal activo. Pues, tal y como afirma Costa, «si esta experiencia ha sido satisfactoria en el plano racional, funcional o emocional, tal vez incluyamos esta marca en nuestro mundo personal de fidelidad a la marca» ${ }^{43}$.

\section{La comunicación de experiencias de marca a través de Facebook: los pa- trocinadores en el campeonato de la Copa del Mundo de Fútbol 2010}

\subsection{Datos iniciales sobre los Patrocinadores del Mundial de Sudáfrica 2010 y de la Selección Española de Fútbol}

El Campeonato Mundial de Fútbol 2010, XIX edición de la Copa Mundial de Fútbol, se celebró en Sudáfrica entre el 11 de junio y el 11 de Julio. Desde el momento en que se supo que Sudáfrica sería la anfitriona para la celebración del Mundial, el país realizó una inversión millonaria para poder estar en condiciones de recibir uno de los eventos deportivos más importantes del planeta.

Previo inicio del campeonato, algunos directivos y representantes de la FIFA parecían estar satisfechos con la cifra publicitaria alcanzada y auguraban que sería el evento deportivo con mayor éxito desde los inicios de su celebración. Lo cierto es que los contratos que se firmaron estaban valorados en más de 820 millones de dólares, cifra que superaba con creces los 700 millones del Mundial de $2006^{44}$.

Siendo la primera vez que el continente africano acogía este evento, los anunciantes nacionales también tenían posibilidades que no habían tenido hasta ahora, ya que la formula publicitaria de los patrocinadores está diseñada del siguiente modo: (A) Socios FIFA: aquellos patrocinadores oficiales y permanentes de la FIFA; (B) Patrocinadores de la Copa Mundial de la FIFA: patrocinadores de cada Copa del Mundo elegidos cada cuatro años; (C) Promotores nacionales: aquellos patrocinadores del propio país de acogida del evento. A estos tres grupos añadimos

42 La comunicación crossmedia consiste en el uso de distintos canales de comunicación que sintonizados los unos con los otros posibilitan una oferta intermedial de productos o servicios; se ofrece una imagen unitaria de la empresa por diferentes medios. Cfr. OlAMENDI, G.: «Crossmedia», Esto es marketing, http://www.estoesmarketing.com/Comunicacion\%20alternativa/Cross\%20Media.pdf. Web visitada el 20/11/2010.

43 CostA, op. cit., 24.

44 «Mundial 2010: desafío Sudáfrica», BBCMundo.com, 7 de agosto de 2006, http://news.bbc.co.uk/go/pr/fr//hi/spanish/business/newsid_5160000/5160132.stm. Web visitada el 05/08/2010. 
un cuarto: (D) Los patrocinadores y colaboradores de la Selección de cada país, aunque para el estudio tendremos en cuenta los patrocinadores de la Selección Española, ya que interesa comprobar y comparar las estrategias y experiencias de marca realizadas por anunciantes tanto locales (en este caso, España, que forma parte de la muestra por cuestiones de cercanía) como globales.

Tabla 1: Relación de patrocinadores del Mundial de Sudáfrica $y$ de la Selección Española ${ }^{45}$

\begin{tabular}{|c|c|c|c|}
\hline PATROCINADOR & $\begin{array}{c}\text { ¿Presencia } \\
\text { en } \\
\text { Facebook? }\end{array}$ & $\begin{array}{l}\text { Número de } \\
\text { seguidores }\end{array}$ & $\begin{array}{l}\text { ¿Experiencia de mar- } \\
\text { ca? }\end{array}$ \\
\hline \multicolumn{4}{|c|}{ SOCIOS PERMANENTES FIFA (6) } \\
\hline Adidas* & $\mathrm{Si}$ & 5.369 .108 & $\begin{array}{c}\text { Three Stories } \\
\text { The Quest / La } \\
\text { búsqueda }\end{array}$ \\
\hline Coca Cola & $\mathrm{Si}$ & 16.611 .286 & Celebraciones \\
\hline Emirates & $\mathrm{Si}$ & 143.509 & No \\
\hline Hyundai Motor & $\mathrm{Si}$ & 93.944 & No \\
\hline Sony & $\mathrm{Si}$ & 285.216 & No \\
\hline Visa & No & -----------. & ------------- \\
\hline \multicolumn{4}{|c|}{ PATROCINADORES FIFA PARA EL MUNDIAL 2010 (8) } \\
\hline Budweiser & $\mathrm{Si}$ & 545.710 & No \\
\hline Castrol* & $\mathrm{Si}$ & 64.442 & World Cup Predictor \\
\hline Continental & $\mathrm{Si}$ & 10.506 & No \\
\hline McDonalds & $\mathrm{Si}$ & 4.627 .519 & No \\
\hline MTN & No & -------------- & -------------- \\
\hline Mahindra Satyam & $\mathrm{Si}$ & 2.417 & No \\
\hline Seara & No & --------------- & --------------- \\
\hline Yingli Solar & No & |-------------- & --------------- \\
\hline \multicolumn{4}{|c|}{ PROMOTORES NACIONALES (6) } \\
\hline $\mathrm{BP}$ & No & -------------- & ------------- \\
\hline Neo Africa & $\mathrm{Si}$ & 227 & One Touch \\
\hline Shanduka-Aggreko & No & -------------- & -------------- \\
\hline FNB & $\mathrm{Si}$ & 1.468 & $\begin{array}{l}\text { Premier Firsts Stadium } \\
\text { Experience } \\
\end{array}$ \\
\hline Prasa & No & -------------- & -------------- \\
\hline Telkom & No & ------------- & --------------- \\
\hline
\end{tabular}

45 Los datos han sido recogidos entre el 01 y el 12 de noviembre del año 2010 con respecto a las publicaciones realizadas durante el periodo comprendido entre el 26 de mayo y el 26 de junio. 


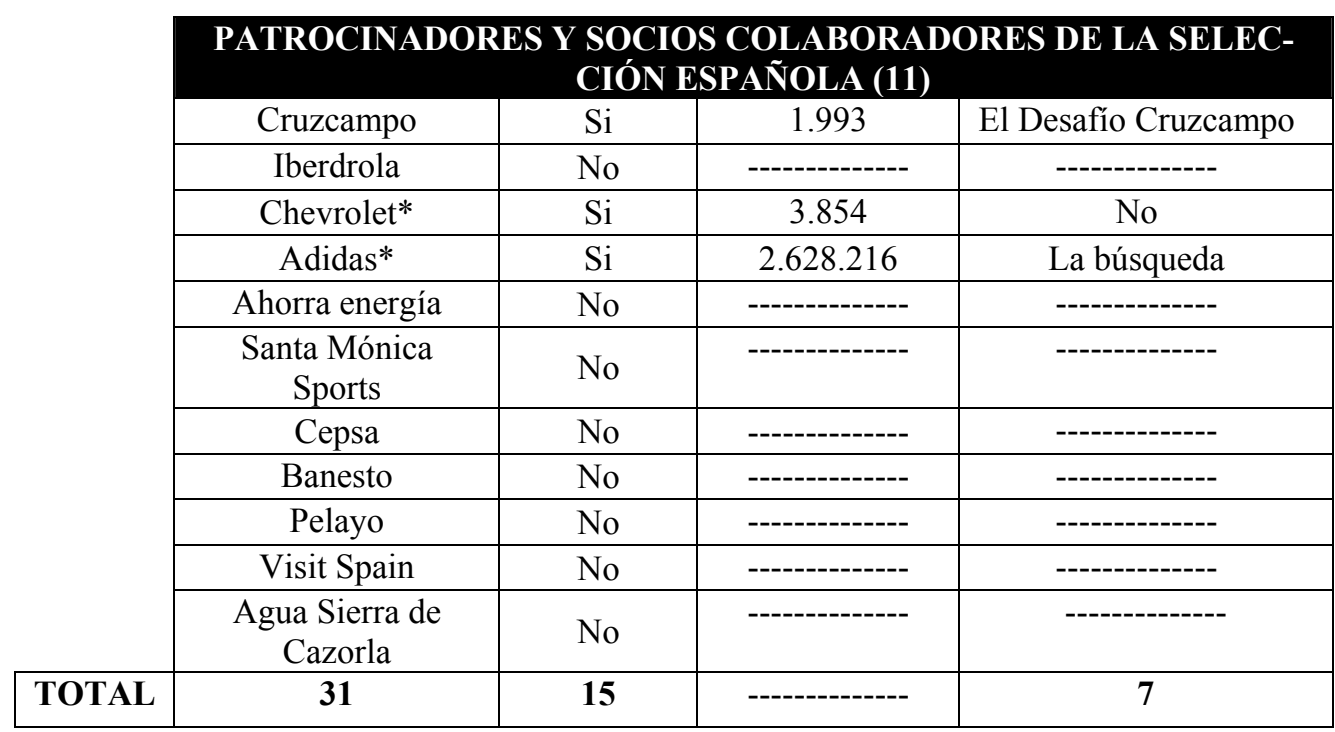

Fuente: Elaboración propia.

* Adidas, a nivel internacional, actúa en la red social a través de su perfil como Adidas Originals; Castrol como Castrol Football (perfil vinculado a su patrocinio en el ámbito deportivo); Chevrolet, a través de uno de sus productos, Chevrolet Spark; y Adidas en España a través de Adidas Football.

Partiendo de la delimitación de esta muestra de estudio, observaremos la presencia de cada uno de estos anunciantes, un total de treinta y uno, en Facebook, analizando las características de dicha presencia, es decir:

a. si dicha presencia es real o simplemente responde a una página informativa creada por la «enciclopedia online» Wikipedia;

b. si es una presencia creada por el propio anunciante, por sus propios fans, por un grupo concreto de consumidores, por el colectivo de trabajadores, etc;

c. si responde a una presencia de tipo corporativa o en función de un producto o marca en concreto de los que posee el grupo anunciante;

d. si tiene creada una página en la red social para la campaña concreta desarrollada como consecuencia de su participación como patrocinador del Mundial.

La delimitación temporal ha sido de un mes exacto, entre el 26 de mayo y el 26 de junio. La idea de concretar así las cuatro semanas largas de observación se debe a la celebración de la fase final del Mundial. Esta se extendió entre el once de junio y el once de septiembre. La intención era tener en cuenta parte de la comunicación durante la celebración del Mundial, pero si considerábamos únicamente el mes de su desarrollo podríamos, de acuerdo con ciertas hipótesis planteadas previamente, 
dejar atrás determinadas campañas cuya principal implantación podría tener lugar antes del inicio del campeonato. Es por ello que era preciso comenzar quince días antes de dicho inicio.

Como podemos comprobar en la Tabla 1, de un total de 31 anunciantes que han ejercido como patrocinadores o socios patrocinadores o colaboradores de la FIFA/Mundial de Sudáfrica/Selección Española de Fútbol -siendo la mayor parte de ellos anunciantes que operan internacionalmente-, solo quince tienen presencia en la red social objeto del estudio, lo que implica aproximadamente el $50 \%$. Esto resulta, cuando menos, trascendente comunicativamente hablando y, en cierto modo, refuta parte de nuestras hipótesis centradas en la inclusión de este tipo de herramientas sociales por la mayor parte de los anunciantes en el contexto publicitario actual. No obstante, algunas de las marcas que figuran en esta muestra no tienen presencia como grupo corporativo, pero sí a través de alguno de sus productos, de otra filial o con otra de sus campañas o promociones. Tal es el caso de Cepsa, que no está presente con un perfil de tipo corporativo ni con una campaña específica de fútbol o para el Mundial de Sudáfrica, pero sí con el «Equipo Competición Cepsa» con el que participan en el Campeonato de Europa FIA de Carreras de Camiones. En lo referente a la no existencia de una filial nos encontramos con el caso de BP, pues figura como uno de los patrocinadores nacionales, por lo que debería figurar BP Sudáfrica y, sin embargo, tan solo muestra su presencia en la red social a través de BP América. Lo mismo sucede con Telkom, otro de los patrocinadores nacionales.

Evidentemente, marcas con presencia a nivel internacional como Adidas, Coca Cola, Sony, Budweiser o McDonalds sí utilizan estas herramientas, ofreciendo información sobre sí mismas y sus productos, tratando de implicar al consumidor con tácticas diversas y utilizando estrategias publicitarias innovadoras que se basan en la sinergia en la Red (conduciendo al consumidor de la red social a otras páginas, así como a la web propia del anunciante). Pero también marcas españolas como Cruzcampo consiguen impresionar al usuario de Facebook llevando a cabo campañas impactantes donde la red social es la clave (hablamos de «El Desafío Cruzcampo»).

Resulta relevante destacar, asimismo, cómo la red social se convierte en reflejo de lo que sucede en la vida real. La aceptación e identificación que los consumidores tienen con una marca se representa de forma virtual en las redes sociales mediante el seguimiento de su perfil y convirtiéndose en partícipes de sus actuaciones.

Por último, otro de los datos significativos que podemos extraer de esta primera observación es que, de esos quince anunciantes con presencia en Facebook, únicamente siete han desarrollado una campaña basada en la experiencia de marca para los consumidores, buscando la participación e implicación con la misma por parte de estos. De nuevo observamos cómo esta cifra se configura en torno al $50 \%$ de aquellos anunciantes que tienen presencia en Facebook, aunque la cifra se reduciría al $22,5 \%$ si tenemos en cuenta el total de la muestra. Estos datos conducen a señalar que no todos los anunciantes muestran su preocupación por el desarrollo de 
brand experiences, por ofrecer una experiencia con atractivo y que resulte inolvidable para el consumidor, lo que no guarda relación con el tipo de producto.

Debemos tener en cuenta que la configuración de estas campañas en la red social se lleva a cabo a través del empleo de fórmulas diversas, y no siempre se utiliza el mismo perfil de la marca para comunicar las experiencias de marca o su presencia publicitaria en un ámbito concreto. En relación con esta conclusión debemos destacar el caso de Cruzcampo, que no posee una presencia directa en Facebook bajo su propia denominación pero sí a través de su campaña para el Mundial «El Desafío Cruzcampo». También Adidas y Castrol crearon perfiles específicos para sus patrocinios relacionados con el mundo del fútbol: Adidas Football y Castrol Football, lo que nos conduce a diferenciar las posibilidades tan heterogéneas que pueden encontrarse en el uso de la red social Facebook desde una perspectiva publicitaria.

\subsection{Análisis de las campañas centradas en experiencias de marca de los patroci- nadores del Mundial de Sudáfrica 2010 y de la Selección Española de Fútbol}

Siguiendo con nuestro proceso de análisis, nos centramos en aquellos patrocinadores del Mundial de Sudáfrica que han desarrollado una campaña basada en la experiencia de marca para el consumidor, a saber: Adidas y Coca Cola dentro del grupo de los socios permanentes de la FIFA; Castrol en el grupo de los patrocinadores de la FIFA para el Mundial 2010; Neo Africa y FNB en el grupo de patrocinadores nacionales; y Cruzcampo y Adidas España en el grupo de los patrocinadores de la Selección Española de Fútbol.

Adidas no solo ha sido patrocinador de la FIFA, sino titular de licencia oficial de la Copa Mundial de la FIFA $2010{ }^{46}$ y también lo será en la de 2014. La marca deportiva desplegó sus esfuerzos publicitarios principalmente a través de dos campañas de experiencia de marca: «Three Stories» y «The Quest» («La Búsqueda» en España). «Three Stories» ha consistido en una serie de actividades, incluyendo el visionado privado de los partidos del Mundial en el $N^{\circ} 66$ de Albert Road, un edificio industrial de sesenta años de antigüedad, en Woodstock, Cape Town. A lo largo de un mes (desde le 11 de junio al 11 de julio) se programaron diversos eventos, entre ellos, una pista interior de skate, exposiciones («Paper Girl», «One Night Only», «The Bin» y «The T-Shirt»), DJs en directo, etc. Por otra parte, «The Quest» ha implicado la creación de una campaña interactiva cuya evolución estaba directamente relacionada con el desarrollo del torneo. «La Búsqueda» es la culminación de una campaña a largo plazo que posiciona a los mejores jugadores del mundo y sus habilidades para formar el mejor equipo. La campaña pregunta «¿Quién llevará a su equipo a la victoria?»e invita a los seguidores del Mundial a interactuar y conversar con otros fans a través de una plataforma múltiple digital.

46 Cfr. Información sobre este patrocinador en la página web corporativa de la FIFA http://es.fifa.com/aboutfifa/marketing/partners/adidas.html. 
La campaña, creada por Adidas y 180Amsterdam/RIOT, incluye 32 jugadores de las 32 federaciones jugando en la Copa Mundial FIFA 2010, pero su inicio ya se remonta a octubre del 2009 , a través de la presentación de un viaje ficticio protagonizado por Zidane en el que trata de reunir a los jugadores más extraordinarios y lograr así el equipo definitivo. Adidas busca con esta campaña mitificar a sus jugadores VIP mediante historias fantásticas sobre su proveniencia e incorporando a cada jugador superpoderes ${ }^{47}$.

The Coca Cola Company, por su parte, es uno de los socios corporativos más antiguos de la FIFA. Su asociación se remonta a 1974 y su primer patrocinio de la Copa Mundial de la FIFA al año $1978^{48}$. Coca Cola se ha anunciado en los estadios de todas las Copas Mundiales de la FIFA desde 1950. Su campaña se basó en el concepto de la «Celebración», y consistió en una ampliación de la actual plataforma de la marca Coca Cola «Destapa la Felicidad», en la que se invita a los aficionados a expresar su pasión por el fútbol a través de diversos tipos de celebraciones incluyendo el baile como protagonista. La campaña ha incluido: La Gira del Trofeo de la Copa Mundial de la FIFA, el himno de K'Naan (un artista de hip-hop de origen somalí y con proyección internacional), la celebración más larga, el Premio Coca-Cola a la mejor celebración, varios anuncios («La historia de la celebración», «Búsqueda» y «Fronteras»), el documental «Coca Cola Presenta: La Celebración de los Goles», una imagen corporativa específica para el Mundial de Sudáfrica 2010, el campamento de Fútbol Coca Cola, los abanderados Coca Cola, etc.

En el grupo de los patrocinadores de la FIFA específicos para el Mundial 2010 únicamente Castrol mantiene presencia en Facebook y ha comunicado a través de la red social su experiencia de marca, el «World Cup Predictor», un plan de apuestas sobre los partidos del Mundial. También ha llevado a cabo otras campañas, como la «Castrol 5A Side»-unos torneos de fútbol previos al Mundial cuya finalidad consiste en la celebración de la asociación de la marca con la FIFA y el Mundial de Sudáfrica y en el que se ofrecen como premios entradas para los partidos-, y otra campaña centrada en la Responsabilidad Social Corporativa (RSC) denominada «Castrol Skillz Holiday Programme», en colaboración con la ONG Grassroots Soccer y que se centra en informar y educar sobre la prevención del SIDA a los más pequeños a través del fútbol. Campañas ambas de las que no ha habido indicios en Facebook durante el periodo de muestra. Con dicho acuerdo ${ }^{49}$, la marca

47 AlvarAdO, V.: «Franz Beckenbauer y Adidas presentan La Búsqueda», Victoralvarado.com, $\mathrm{http}: / /$ victoralvarado.com/2010/06/08/franz-beckenbauer-y-adidas-presentan-la-busqueda. Web visitada el 08/11/2010.

48 Cfr. Información sobre este patrocinador en http://es.fifa.com/aboutfifa/marketing/partners/cocacola.html.

49 Para este acuerdo ha sido relevante el «Índice de Rendimiento», una herramienta desarrollada por Castrol para la Eurocopa del 2008 mediante la que los aficionados al fútbol podían analizar, de forma objetiva, la actuación individual de los jugadores y de las selecciones. Una experiencia futbolística de gran 
global de lubricantes consiguió los derechos de patrocinio en todo el mundo para la Copa del Mundo FIFA World Cup de Sudáfrica 2010, la Copa del Mundo FIFA World Cup de Brasil 2014 y las dos Copas Confederaciones de la FIFA que se celebrarán entre los años 2007 y 2014.

Ya en la categorización de los patrocinadores nacionales, son FNB y Neo Africa los representantes por ser los únicos que tienen presencia en Facebook y, al mismo tiempo, idearon una campaña centrada en la experiencia de marca en la que trataron de implicar al usuario de la red social. Neo Africa -a pesar de que su campaña con mayor repercusión fue «Sing it Loud, Sing it Proud», un concurso que tenía como tema central la interpretación del himno nacional de Sudáfrica-, ofreció la implicación de sus consumidores reales o potenciales a través de la campaña de experiencia de marca «One Touch». «One Touch», basada en un nuevo concepto en el mundo del deporte que se introdujo en todos los FIFA Fan Fests de Sudáfrica, proporcionando a los niños menores de dieciséis años actividades de aprendizaje y construcción, mediante un juego centrado en el uso del balón. La participación en esta campaña, por tanto, ha sido la clave para su desarrollo y éxito.

El otro socio nacional que presentó una campaña de este tipo ha sido First National Bank (FNB). FNB aceptó el rol de ser el Banco Oficial sudafricano de la FIFA, proporcionando todo tipo de servicios de banca y transacciones durante las competiciones de la Copa Confederaciones de la FIFA del año 2009 y el Mundial del 2010. El patrocinio incluía un paquete de derechos publicitarios, promocionales y de marketing para ambas competiciones en la categoría de productos financieros con Sudáfrica. No obstante, su experiencia de marca con los seguidores del Mundial se centró en la campaña «Premier First Stadium Experience», que ofrecía la oportunidad de conocer y disfrutar diversas experiencias en los estadios oficiales del Mundial.

En España, las marcas que mejor han sabido vender su presencia en el Mundial asociadas a la Selección Española de Fútbol han sido precisamente aquellas que ofrecían una campaña de experiencia de marca: Adidas y Cruzcampo, tal como lo señala el «Estudio Sindicado del Mundial y Selección de Fútbol» realizado por Synovate ${ }^{50}$. La campaña de Cruzcampo consistió en una acción centrada en la participación y el voluntariado de los aficionados al fútbol y fans de la Selección Española. La idea era llevar un balón, el Balón de la Afición, desde Madrid hasta Johanesburgo (aproximadamente

alcance, con la que Castrol dio vida a los principales valores de la marca, tales como la pasión, el avance tecnológico y el rendimiento.

50 En este estudio se ofrecen ciertos resultados acerca de la mejor gestión de la colaboración entre marcas y Selección para el consumidor. De acuerdo con este estudio, son Adidas y Cruzcampo las marcas que ocupan el primero y segundo lugar, respectivamente, con poco más de un punto de diferencia, en el top 10 de marcas asociadas a la Selección Española como patrocinador. Además, el conocimiento espontáneo de la marca patrocinadora aumentó de forma considerable una semana después de la victoria del Mundial por parte de la Selección, siendo destacable el caso de Cruzcampo que creció 13'4 puntos con respecto al inicio del Mundial. Cfr. «Cruzcampo y Adidas, las marcas que mejor supieron gestionar su patrocinio a la Selección», Marketingdirecto.com, http://www.marketingdirecto.com/actualidad/anunciantes/cruzcampo-yadidas-las-marcas-que-mejor-supieron-gestionar-su-patrocinio-a-la-seleccion/. Web visitada el 5/11/2010. 
8.000 kilómetros), gracias a la colaboración de miles de voluntarios en un trayecto que duró más de cuatro semanas (desde el 24 de abril al 12 de junio). La acción fue bautizada por la marca como un «símbolo de apoyo de todo el país». Una vez en Sudáfrica, el balón fue entregado por el «Delegado de la Afición»a a la Selección Española de Fútbol y a su entrenador, Vicente del Bosque.

En cuanto a la importancia de estas acciones en la red social, hemos delimitado el análisis de ciertos parámetros que ayudan a entender la relación entre experiencia de marca y red social en base al comportamiento de la marca en su perfil de Facebook. Estos parámetros son los siguientes: número total de publicaciones que ha realizado la marca en el periodo temporal propuesto, número de publicaciones que referencian el Mundial de Sudáfrica (los partidos, su desarrollo, noticias relacionadas con el evento deportivo, búsqueda de opiniones sobre determinados resultados, alineaciones, jugadas, etc.), número de publicaciones en el perfil de la marca que tienen como base ofrecer información o pedir participación para la campaña de brand experience y, ya por último, el número de comentarios de cada una de estas publicaciones.

Tabla 2: Referencias en Facebook a la campaña de experiencia de marca

\begin{tabular}{|c|c|c|c|c|c|}
\hline Marca & $\begin{array}{l}\text { Experiencia } \\
\text { de marca }\end{array}$ & $\begin{array}{c}\mathbf{N}^{0} \\
\text { total } \\
\text { public. }\end{array}$ & $\begin{array}{l}\mathbf{N}^{\mathbf{0}} \text { pu- } \\
\text { blic. } \\
\text { Mundial }\end{array}$ & $\begin{array}{l}\mathbf{N}^{0} \text { public. } \\
\text { exp. de } \\
\text { marca }\end{array}$ & $\begin{array}{l}\mathbf{N}^{0} \text { comentarios } \\
\text { por publicación }\end{array}$ \\
\hline Adidas & $\begin{array}{l}\text { Three Stories } \\
\text { The Quest / La } \\
\text { Búsqueda }\end{array}$ & 55 & $\begin{array}{c}18 \\
(32,73 \%)\end{array}$ & $\begin{array}{l}3 \\
1\end{array}$ & $\begin{array}{c}122,16,76 \\
163\end{array}$ \\
\hline Coca Cola & Celebraciones & 13 & $7(53,85 \%)$ & 6 & $\begin{array}{c}157,224,197,201, \\
200,172\end{array}$ \\
\hline Castrol & World Cup Predictor & 43 & $\begin{array}{c}33 \\
(76,74 \%)\end{array}$ & 26 & $\begin{array}{c}70,105,80,93,157, \\
52,69,66,76,34, \\
26,24,35,38,41 \\
31,30,74,15,20, \\
15,23,14,55,68,51\end{array}$ \\
\hline Neo Africa & One Touch & 10 & $9(90 \%)$ & 5 & $1 / 1 / 2 / 2 / 1$ \\
\hline FNB & $\begin{array}{c}\text { Premier Firsts Stadium } \\
\text { Experience }\end{array}$ & 9 & $5(55,56 \%)$ & 1 & 4 \\
\hline Cruzcampo & El Desafío Cruzcampo & 107 & $\begin{array}{c}92 \\
(85,98 \%)\end{array}$ & 21 & \begin{tabular}{|c|}
$12,4,8,2,2,0,15$ \\
$14,10,1,10,2,7,5$ \\
$5,2,8,5,3,1,9$ \\
\end{tabular} \\
\hline $\begin{array}{c}\text { Adidas } \\
\text { (Football) }\end{array}$ & $\begin{array}{l}\text { The Quest / La Búsque- } \\
\text { da }\end{array}$ & 96 & $\begin{array}{c}82 \\
(85,42 \%)\end{array}$ & 30 & $\begin{array}{l}43,14,33,43,31 \\
29,54,13,24,36 \\
15,29,25,20,8,9 \\
23,9,38,15,7,35 \\
18,25,5,29,26,34 \\
3,40\end{array}$ \\
\hline
\end{tabular}

Fuente: Elaboración propia. 
Tras la recopilación de estos datos, podemos extraer algunas conclusiones interesantes en torno al uso que cada una de estas marcas -diversas en cuanto a sector, tamaño y amplitud geográfica en la que operan- realizan de las redes sociales. Para empezar, en cuanto al número de publicaciones que la marca realiza, de forma genérica, en su perfil de Facebook, nos topamos con que las que menos importancia otorgan a la comunicación a través de esta herramienta son, por este orden, FNB, Neo Africa y Coca-Cola. Sorprende que una marca con altos niveles de aceptabilidad y sociabilidad como es Coca-Cola se encuentre en esta posición en cuanto a su actuación a través de la red social Facebook. Por el otro lado, las marcas que mayor relevancia otorgan a este tipo de comunicación son Cruzcampo (a través de su perfil «El Desafío Cruzcampo» creado en exclusiva para el Mundial de Sudáfrica), Adidas (Football), Adidas (Originals) y Castrol. La empresa cervecera española, de la mano de la agencia publicitaria Ogilvy \& Mather, lidera así este ranking mostrando su aceptación de las NTICs aplicadas al ámbito comunicativo.

El siguiente dato se relaciona con la implicación de la marca con el Mundial de Sudáfrica 2010. Así, debemos realizar una valoración porcentual en cuanto a la relación entre número de publicaciones totales en el perfil de Facebook y el número de publicaciones con contenido sobre este Campeonato para entender la relevancia relativa y no únicamente en cifras totales. De este modo descubrimos que el anunciante que mayor implicación ha mostrado para con el Mundial ha sido Neo Africa $(90 \%$ de publicaciones sobre el total), seguida de Cruzcampo $(85,98 \%)$, Adidas Football $(85,42 \%)$ y Castrol $(76,74 \%)$. Las que menos relevancia le han otorgado a publicar información relacionada con el Campeonato de la FIFA han sido Adidas (con un $32,73 \%$ ), Coca-Cola $(53,85 \%$ ) y FNB $(55,56 \%)$.

En cuanto a la relevancia otorgada a la comunicación de la experiencia de marca diseñada para el Mundial, las marcas que destacan, y con creces, son: Adidas Football con su campaña «The Quest»//«La Búsqueda» (30 entradas), Castrol con «World Cup Predictor» (26 entradas) y, por último, Cruzcampo con «El Desafío Cruzcampo» (21 entradas). Mientras, en el lado opuesto, figuran FNB (con una única publicación), Adidas (también con una única entrada sobre la campaña «The Quest» y tres entradas para «Three Stories»), Neo Africa (con 5 entradas) y CocaCola (con 6).

El último de los parámetros que se va a analizar versa sobre el número de comentarios que los usuarios de la red social y seguidores del perfil de estas marcas han realizado en cada una de las publicaciones sobre las brand experiences. Las campañas de Adidas, Coca-Cola y Castrol son las que provocan la mayor intervención por parte de los usuarios de la red social. Parece que conceptos como la celebración, la intriga por deducir los resultados de los partidos en una competición o la organización de determinadas «fiestas» con apoyo en la música, el arte y el deporte resultan buenas fórmulas para acercarse a los consumidores.

Para finalizar, y teniendo en cuenta un cruce de las variables analizadas (realizando una suma de los puestos que finalmente han ocupado estos anunciantes en el 
estudio de los parámetros delimitados), concluimos que las dos marcas con el desarrollo de una brand experience más significativa y exitosa en su desarrollo virtual a través de redes sociales han sido Cruzcampo con «El Desafío Cruzcampo» y Adidas (Football) con «La Búsqueda», en su perfil de Facebook para España y en exclusiva para el sector deportivo.

\section{Conclusiones}

Tras la elaboración de este artículo podemos establecer ciertas ideas conclusivas. Para empezar, consideramos que la experiencia de marca es vivida por unos y por otros de forma diferente, pero de lo que no cabe duda es que es pensada por agencias y anunciantes como una fórmula para lograr que el consumidor deposite su confianza en la marca. Es por ello que lo más importante de una estrategia de este tipo reside en los beneficios que obtiene el consumidor y, en consecuencia, deben ser cuidados, principalmente, aspectos cómo los valores qué se transmiten, la forma de participación que se ofrece al usuario/consumidor y cómo se obtiene su confianza.

Por otra parte, la presencia de las empresas en las redes sociales se utiliza como un lugar informativo y de intervención para los consumidores (tanto con la empresa como entre ellos, estableciéndose como un grupo de individuos con valores comunes) y no como un mero lugar para noticias corporativas de la empresa. Se deben ofrecer contenidos interesantes y atractivos para los usuarios y que aporten valor a los clientes de la empresa. Sobre todo se busca promover la participación, crear una comunidad que reaccione ante las noticias y eventos que se vayan incorporando y que, sobre todo, interactúe con la marca para conseguir una relación más cercana y con una mayor complicidad, estableciendo diálogos directos entre marca y consumidor/usuario de la red social. Es necesario la creación de un ambiente donde la gente se anime a aportar sus ideas e incluso a publicar sus propios contenidos, su propia información. Se trata de la conversión del consumidor en prosumidor donde la reciprocidad es la esencia.

No obstante, la movilización del usuario dependerá del grado de atractivo que tenga la campaña, la propuesta y lo que se le ofrece. Es por ello que, para incentivar inicialmente esta participación, las agencias crean falsos usuarios, aunque, evidentemente, con el análisis realizado no podemos determinar si en las campañas que conforman la muestra fueron empleados este tipo de recursos.

Teniendo estas ideas en cuenta y la exploración que se ha desarrollado, refutamos ciertas hipótesis que nos habíamos planteado con la elaboración de este artículo, pues lo cierto es que el empleo de brand experiences no está tan extendido todavía, al menos en lo que se refiere a su aplicación a un evento concreto que ofrece amplias posibilidades desde una perspectiva publicitaria, como ha sido el caso del Mundial de Fútbol de Sudáfrica. Los patrocinadores de este campeonato han optado por campañas más convencionales mediante el empleo de spots televisivos y 
otro tipo de presencia promocional no audiovisual, pero que no buscaban en último término la implicación del consumidor ni tampoco se planteaban el logro de una relación más próxima y directa, pues a este fin ayudaría en gran medida el empleo de las redes sociales.

Sin embargo, aquellos anunciantes que sí utilizan las redes sociales como fórmula comunicativa se apoyan en crear brand experiences o piezas con las que, en todo caso, pretenden conseguir una respuesta de su target en un plazo de tiempo muy corto, diseñando proyectos creativos que evolucionan en su desarrollo temporal. Las agencias deben prever las posibles circunstancias a las que se tendrá que enfrentar la campaña para ir adaptándose a un nuevo contexto que resulta imposible plantearse de forma cerrada desde su planificación. Pero he aquí lo interesante y novedoso de la utilización de estas herramientas que favorecen las relaciones sociales, cualidades que se intensifican en su aplicación a un contexto publicitario complejo e imprevisible en su desarrollo como es el caso de un campeonato deportivo.

A pesar de que ciertas afirmaciones planteadas en el inicio de este artículo se vienen abajo, otras ideas sí se confirman, como es el caso del interés que muestran los usuarios de la red social por el seguimiento de marcas concretas, así como por aumentar su sentimiento de sentirse partícipes de lo que la marca en cuestión comunica, comentando, exponiendo ideas, debatiendo o expresando su apoyo ante determinadas campañas con las que se sienten identificados. Su contribución se configura como el principal símbolo de lo que se entiende por la realización de una campaña a través de las redes sociales, al mismo tiempo que una experiencia de marca en sí misma en la que, si el consumidor obtiene respuesta ante sus dudas o comentarios, se generará un aumento de su confianza en la marca y predisposición para recibir información sobre ella y sus productos. De ahí que se incentiven este tipo de comportamientos a través de promociones con premios de lo más diverso (aunque en este caso todos guardaban relación con el mundo futbolístico y, más concretamente, con el Mundial). La clave es la comunicación, una comunicación real aunque en un mundo virtual, lo que parece una paradoja, pero que no deja de ser uno de los beneficios que la red y sus innumerables herramientas han incorporado al ámbito empresarial de forma global y, más concretamente, al publicitario.

En consecuencia, podemos delimitar que este tipo de campañas sí tienen como finalidad la búsqueda de participación del usuario, pero no solo una respuesta de tipo emocional, tal y como señalábamos en el planteamiento de hipótesis iniciales, sino también réplicas y comportamientos racionales a través del debate, de ideas y consultas que permitan al anunciante y a las agencias la obtención de una base sólida en torno a los intereses y forma de pensar del consumidor sobre la que determinar futuras campañas o cambios sobre los proyectos ya en marcha. Más que comunicación, las redes sociales también se configuran, por tanto, en estudios de tipo sociológico y en investigación de mercados, con datos aplicables y de los cuáles se debe hacer uso para conseguir crear futuras experiencias de marca atrayentes para el consumidor. 


\section{Fuentes de consulta}

AlLEGRO 234: «Los resultados del Coolest \& Gaps Branding Survey», web corporativa de la empresa Allegro 234: Strategic Marketing and Branding, http://www.allegro234.net/noticia.asp?vcnoticia=14.

AlvarADO, V.: «Franz Beckenbauer y Adidas presentan La Búsqueda», Victoralvarado.com, http://victoralvarado.com/2010/06/08/franz-beckenbauer-y-adidas-presentan-labusqueda.

AimC-Asociación Para La Investigación De Los Medios De Comunicación (2010): Navegantes en la Red. $12^{a}$ encuesta AIMC a usuarios de Internet, Madrid, Sersa.

ASOCIACIÓN ESPAÑOLA DE AGENCIAS DE PUBLICIDAD: «Estudio de Agencias 2008. Estudio de Imagen de las Agencias de Publicidad en España» (versión resumen online). http://www.aeap.es/archivo/e01816537c5196080e1c3ee181130eae.pdf.

Christakis, N. A.; Fowler, J. H. (2010): Conectados, Madrid, Taurus.

CostA, Joan (2004): La imagen de marca, Barcelona, Paidós.

FAERMAN, J. (2010): Faceboom. Facebook, el nuevo fenómeno de masas. Barcelona, Ediciones B Argentina y Grupo Planeta.

HOLBROOK, M. B.; HIRSCHMAN, E. C. (1982): «The experiential aspectos of consumption: consumer fantasies, feelings and fun», The Journal of Consumer Research», 9(2), Chicago, The University of Chicago Press, 132-140.

LASAlle, D.; BRITTON, T. A. (2006): Cómo añadir valor a sus productos y servicios: convertir los productos ordinarios en experiencias extraordinarias, Barcelona, Deusto.

LI, C.; Bernoff, J. (2008): El mundo Groundswell. Cómo aprovechar los movimientos sociales espontáneos en la Red, Barcelona, Ediciones Urano.

Lindstrom, M. (2007): Brand sense. Convierta su marca en una experiencia de cinco sentidos, México, Grupo Editorial Patria.

LÓPEZ Vidales, N.; Peñafiel SaIZ, C. (coordinadoras y editoras) (2003): Odisea 21. La evolución del sector audiovisual. Modos de producción cambiantes y nuevas tecnologías, Madrid, Editorial Fragua.

Montero, L.: «La experiencia de marca», Baquia: Negocios y Nuevas Tecnologías, http://www.baquia.com/com//20021211/art00011.html.

OLAMENDI, G.: «Crossmedia», Estoesmarketing.com, http://www.estoesmarketing.com/Comunicacion\%20alternativa/Cross\%20Media.pdf.

PInE, B. J.; GILMORE, J. H. (1999): The Experience Economy: Work is theatre and every Business a stage, Boston, Harvard Business School Press.

REQUENA SANTOS, F. (1989): «El concepto de red social», Revista de Investigaciones Sociológicas-REIS, 48, 137-152.

Royo, J.: «Identidad líquida. Las marcas en el ciberespacio», Joancosta.com, http://www.joancosta.com/identidad_liquida.htm.

SARACCO, C.: «La experiencia de marca va más allá de un símbolo», Business Opportunities, http://espanol.business-opportunities.biz/2006/02/28/la-experiencia-de-marca-vamas-alla-de-un-simbolo/.

SCHMitT, B. (1999): Experiential Marketing: how to get customers to sense, feel, think, act and relate to your company and brands, Nueva York, The Free Press. Trad. esp. Deusto, Barcelona, 2006. 
SimMEL, G. (1986): Sociología, 1. Estudios sobre las formas de socialización, Madrid, Alianza Editorial.

UGARTE, D. (2007): El poder de las redes. Manual ilustrado para personas, colectivos y empresas abocados al ciberctivismo, Barcelona, El Cobre Ediciones.

«Cómo transmitir la experiencia de marca a través de la arquitectura», web corporativa de la la revista de decoración Espacioliving.com, http://www.espacioliving.com/nota.asp?nota_id=1223734.

«Cruzcampo y Adidas, las marcas que mejor supieron gestionar su patrocinio a la Selección», Marketingdirecto.com, http://www.marketingdirecto.com/actualidad/anunciantes/cruzcampo-y-adidas-lasmarcas-que-mejor-supieron-gestionar-su-patrocinio-a-la-seleccion/.

«Mundial 2010: desafío Sudáfrica», BBCMundo.com. 7 de agosto de 2006, http://news.bbc.co.uk/go/pr/fr/-/hi/spanish/business/newsid_5160000/5160132.stm.

Página web corporativa de Camper http://www.casacamper.com/barcelona/default-es.html. Página web corporativa de la FIFA http://es.fifa.com/aboutfifa/marketing/partners. 